\title{
Defect chemistry in ferroelectric perovskites: long standing issues and recent advances
}

\author{
M. Maglione *, G. Philippot , D. Levasseur , S. Payan , C. Aymonier and C. Elissalde
}

CNRS, Univ. Bordeaux, ICMCB, UPR 9048, F-33600, Pessac, France.

E-mail: mario.maglione@icmcb.cnrs.fr

\begin{abstract}
:
Accurate control of residual defect density is required for reliable investigation and use of ferroelectric materials. After reviewing the long term endeavor to decrease defect contributions in bulk materials, which reached mass production decades ago, recent challenges are underlined. These mostly result from the continuous trend towards integration which has reached the nanometre range. The contribution of solid state chemistry is of key relevance for improving the present processing routes and suggesting alternative ones, for example by controlling a large density of charged defects to reach unprecedented functionalities. Some of these breakthroughs are reviewed.
\end{abstract}

\section{Introduction}

Residual point defects are relevant in any material but they have very specific contributions in the case of ferroelectric compounds. Indeed, ferroelectricity and the related properties like piezoelectricity or pyroelectricity result from a long range correlation among ionic displacements in polar lattices as exemplified in Fig. 1 for the case of $\mathrm{BaTiO}_{3}$, the archetypical ferroelectric compound. In this perovskite, which is the most investigated and used ferroelectric compound, the parent non-ferroelectric cubic phase transforms into a ferroelectric one, when all $\mathrm{Ti}^{4+}$ ions located initially at the centre of the oxygen octahedra go off-centre in a long range process which is coherent over macroscopic length scales. Such off-centring takes place successively along $\langle h 00\rangle,\langle h h 0\rangle$ and $\langle h h h\rangle$ crystallographic directions thus leading to the phase transitions sequence cubic-tetragonal-orthorhombic-rhombohedral in cooling. In all of the three ferroelectric phases, the key parameter which is the polarization resulting from the $\mathrm{Ti}^{4+}$ off-centring, thus intimately results from a structural transformation of the perovskite lattice. There is a one-to-one interplay between the functionality and the structure thus raising the issue of all structural defects as a source of perturbation for the polarization. Related properties like dielectric permittivity, which probes the sensitivity of this polarization to an external electric field, is also very sensitive to lattice defects. In the specific case of point defects, which is the focus here, not only the size and mass of the defects, but also the chemical binding and the valence directly disturb the properties. All these factors also matter in conducting, semi-conducting and magnetic materials through the contributions of point defects to electronic excitations but the link with structural transformation is not as direct as in ferroelectrics.

It is the aim of the present paper to describe examples of point defects and their influence on dielectric properties, restricting ourselves to $\mathrm{BaTiO}_{3}$-based materials. The first case to be taken up is oxygen vacancies which are unavoidable deficiencies of the $\mathrm{ABO}_{3}$ perovskite. Beyond the direct effect of electron donors, which raises the conductivity, increasing their density leads to macroscopic space charge which artificially increases the dielectric permittivity. While this is well established in bulk single crystals and 
ceramics, recent advances were achieved to tune such space charges in nanostructured ceramics. Two of these are discussed, namely the mechano-synthesis and the core-shell approach. While being unavoidable, oxygen vacancies can be compensated under substitution of $\mathrm{Ti}^{4+}$ cations by electron acceptors like, for example, manganese. This is again not a new concept for ceramics and single crystals but has been recently used for thin films leading to unambiguous description of the electron trapping mechanism which is not always the case in bulk materials. We then address the case of -OH radicals which are of increasing influence in soft chemistry processed nano-sized powders. Advanced routes like the supercritical one were shown to be useful in distinguishing the various types of substitution of $\mathrm{OH}-$ radicals in the bulk or at the surface of the nano-grains. This is of key relevance when probing the polarization at nanoscale using local probes. Tracking charged defects in ferroelectric thin films and nanopowders is also a critical issue for the use and reliability of integrated microelectronic components for information storage and telecoms.

While all the above charged defects are of low density, from ppm up to a few percent, heavy substitution of isovalent cations can deeply change the polarization without increasing the conductivity. This has been already applied in $\mathrm{PbTiO}_{3}-\mathrm{PbZrO}_{3}(\mathrm{PZT})$, the reference piezoelectric material which has the highest performance close to the $50 / 50 \mathrm{Zr} / \mathrm{Ti}$ substitution range. While broadly used for ultrasonic sensors and actuators, PZT has the obvious drawback of containing lead, a spurious chemical, whose replacement is required. Towards this aim, the last part of this paper is about simultaneous substitution on the $\mathrm{Ba}$ and $\mathrm{Ti}$ sites in $\mathrm{BaTiO}_{3}$ leading to a full solid-solution in the ternary $\mathrm{BaTiO}_{3}-\mathrm{CaTiO}_{3}-\mathrm{BaZrO}_{3}$ diagram in which extremely large piezoelectricity occurs at the point where the three already mentioned phase transitions merge into a single one.

\section{Free charge motion and dielectric properties}

The dielectric permittivity is a key parameter for ferroelectric materials. It is a complex number $\varepsilon^{*}=\varepsilon^{\prime}-$ $\mathrm{j} \varepsilon^{\prime \prime}$, where $\varepsilon^{\prime}$ is the proportionality coefficient which relates the polarization to an external electric field and $\varepsilon^{\prime \prime}$ quantifies the variance from perfect dielectric behaviour. Electronic and ionic conductivity can increase $\varepsilon^{\prime \prime}$, which is strictly zero only for a perfect vacuum; in all materials, it is better quantified by the dielectric losses $\operatorname{tg} \delta=\frac{\varepsilon^{\prime}}{\varepsilon^{\prime \prime}}$ which is a measure of the amount of electric field lost in charge transfer, while it should only increase the polarization. In this paper the mobile charges which increase the imaginary permittivity are only electrons (or holes). Note that sometimes $\varepsilon^{\prime}$ is written as $\varepsilon_{1}$ while $\varepsilon^{\prime \prime}$ is written as $\varepsilon_{2}$. All these experimentally accessible parameters are dependent on frequency, temperature, electric field and many other stresses. The way they depend on these external stresses tells us a lot about their microscopic origin. For example, the real and imaginary parts of the dielectric permittivity change monotonously with frequency and temperature in the case of contributions from mobile charges while they undergo non-monotonous and even sharp anomalies in the case of local ionic motions (like for example the orientation change of polarization among one of the directions in Fig. 1). Ferroelectric materials are largely investigated and produced at industrial scales because of their outstanding dielectric permittivities being between 1000 and 10 000, while other oxides lie in the sub-100 range. This by no way means that all their dielectric properties are the same. Indeed, the dielectric losses - which are a direct measure of the variance from perfect dielectric behaviour - are usually of several percent. This is the first evidence that ferroelectric compounds are not perfect in every respect and that their losses and conductivity may be greatly increased.

\subsection{Vacancies}

The sensitivity of ferroelectric materials to the density of oxygen vacancies is usually probed in reduction experiments where the electronic conductivity of $\mathrm{BaTiO}_{3}$ ceramics is tuned at high temperatures by 
changing the oxygen partial pressure from 0.2 down to $10^{-20} \mathrm{~atm}$. The " $\mathrm{V}$ " type plot which is observed at temperatures higher than ca. $500{ }^{\circ} \mathrm{C}$ demonstrates a cross-over from hole conductivity under moderate reduction to an electron one in the lowest oxygen partial pressures (Fig. 2).. This is readily explained using the Kröger and Vink approach which is used to balance all the charged defect concentrations. $\stackrel{2-5}{ }$ While all are in agreement that oxygen vacancies play a major role in these processes, the way they get compensated by related lattice cationic defects is still a matter of debate. $\frac{6,7}{\mathrm{In}}$ particular the possible occurrence of Ti vacancies at the centre of the oxygen octahedra is a highly efficient compensating centre (one $\mathrm{Ti}^{4+}$ vacancy for two $\mathrm{O}^{2-}$ vacancies). The question remains regarding up to which density the perovskite lattice can stand such defects occurring in the closed octahedral site. Cationic reduction of $\mathrm{Ti}^{4+}$ into $\mathrm{Ti}^{3+}$ or even full $\mathrm{Ba}^{2+}$ vacancies in the opened dodecahedral $\mathrm{Ba}$ site is more likely to occur. Once a given reduction state of $\mathrm{BaTiO}_{3}$ has been reached, not only is the conductivity tuned as seen in Fig. 2 but also the dielectric permittivity is changed to a great extent. In Fig. 3 , the dielectric permittivities of $\mathrm{BaTiO}_{3}$ single crystals are reported after different high temperature annealing steps. One first sees that the 120 ${ }^{\circ} \mathrm{C}$ ferroelectric transition temperature is kept with identical dielectric permittivity while a large and broad maximum increases in highly reduced samples (note the log scale for permittivity in Fig. 3). Such a maximum was explained by the accumulation of free charges at the crystal surfaces leading to the concept of space charge relaxation. $\frac{8-10}{}$ This is the first piece of evidence that the intrinsic lattice polarizability of ferroelectric materials plays a key role in the interplay between reduction-induced free charges and the measured dielectric parameters.

\subsection{Size effect}

Further evidence for the competition between conductivity and polarization is the observed cross-over from the pure ferroelectric behaviour of thick films towards resistive switching in ultrathin films of only a few nanometers thickness. ${ }^{11-14}$ The widely accepted model for such resistive switching is that ionic motion (most probably oxygen) and accumulation in the form of space charges could explain the resistive switching. $\frac{13}{}$ While this has been reported in many ternary and binary oxides, including non-ferroelectric ones, the very specific feature of ferroelectric materials seems to be the strong influence of substrate strain into the resistive switching process. This again shows that ferroelectric net polarisation and free charge motion are inter-related, the free charges being of electronic and/or ionic nature.

\section{Oxygen sub-stoichiometry and ionic conductivity}

Reverting to bulk materials which is the main concern of this paper, full ionic conductivity can be achieved in extremely reduced perovskites. $\underline{15}$ One way to explain both the stabilization of a high density of oxygen vacancies and the oxygen ionic conductivity could be the occurrence of the Ruddlesden-Popper phase at the local level. Such phases are deduced from the compact $\mathrm{SrTiO}_{3}$ lattice by the insertion of SrO planes between the perovskites' units of $\mathrm{Fig}$. 1 leading to the general formula $\mathrm{SrO}\left(\mathrm{SrTiO}_{3}\right)_{\mathrm{n}}$ having the two end members $\mathrm{Sr}_{2} \mathrm{TiO}_{4}$ for $n=1$ and $\mathrm{SrTiO}_{3}$ for $n=\infty$. These phases may display large mobility in the case of non-stoichiometry. Their structural compatibility and easy occurrence within a perovskite network were recently confirmed in two ways. The first was the case of $\mathrm{SrTiO}_{3}$ high quality single crystals in which the Ruddlesden-Popper phase could be induced reversibly by a moderate electric field at the near surface. $\underline{16}$ The coexistence between perovskite and Ruddlesden-Popper was seen through grazing incidence X-ray diffraction, which means that the amount of the latter in the former is far from being negligible when considering the very near surface atomic layers. Besides the electric field, chemical pressure is another useful way for the insertion of $\mathrm{Ba}$ at the near surface of $\mathrm{BaTiO}_{3}$ films thus possibly leading to RuddlesdenPopper phases. This chemical pressure can result from $\mathrm{Nb}^{5+}$ substitution to $\mathrm{Ti}^{4+}$ thus driving the migration of $\mathrm{Ba}^{2+}$ to the outer surface. $\underline{17}$

\subsection{Challenge}


After pioneering experiments on highly reduced non-ferroelectric perovskites, the question remained of how to fix an intermediate reduction level in bulk polycrystalline ferroelectric perovskites. $\underline{15}$

Mechano-synthesis. Many attempts have been made in this direction, which were not easy to reach since re-oxidation spontaneously occurs whenever the reduction induced lattice distortions are not enough to stabilize the charged defects. Two recent routes towards this goal have now been described. The first is the use of mechano-synthesis of a $\mathrm{BaTiO}_{3}-\mathrm{CaH}_{2}$ mixture. $\frac{18}{n}$ The gradual deepening of the overall blue colour of $\mathrm{BaTiO}_{3}$ was taken as evidence from naked eye for the $\mathrm{Ti}^{3+}$ defect concentration gradually increasing as the density of anionic $\mathrm{H}^{-}$defects in the oxygen sites increased up to 20 atm\%. A very interesting outcome is the possible use of $\mathrm{H}^{-}$substituted $\mathrm{BaTiO}_{3}$, originally a dielectric, as a hydrogen storage and conducting material. $\underline{18}$ Unfortunately the stabilization of such very high hydrogen content required moderate sintering temperature thus precluding the densification which is required for investigating the dielectric properties.

Core-shell particles. Another way of obtaining intermediate reduction in $\mathrm{BaTiO}_{3}$ was achieved by using silica coated $\mathrm{BaTiO}_{3}$ grains, so called core-shell particles, and sintering them using advanced and fast Spark Plasma Sintering (SPS). $\underline{19}$ When used under a reducing atmosphere, tuned reduction can be achieved. The model involves the fast sintering step, which lasts a few minutes, over which oxygen can be released by the $\mathrm{BaTiO}_{3}$ core through the viscous $\mathrm{SiO}_{2}$ and re-oxidation is precluded because of the ultrafast cooling step which is analogous to a quenching. In this last step, the re-frozen silica shell recovers a very low oxygen mobility thus acting as a barrier against re-oxidation. As a result, the composite ceramics achieve a gradually deepening blue very similar to the one observed for $\mathrm{H}^{-}$substituted $\mathrm{BaTiO}_{3}$ (Fig. 4). Again, the stable $\mathrm{Ti}^{3+}$ state should be taken into account to explain this colour change. 20 Deeper investigations are needed to confirm this observation. First, the dielectric permittivities of the reduced composites were very much altered as compared to the raw materials. These alterations were twofold:

- The dielectric permittivity was very large $\left(\varepsilon>10^{5}\right)$ and temperature independent over a very large temperature interval $(100 \mathrm{~K}<\mathrm{T}<400 \mathrm{~K})$. This behaviour, which is against the Curie $1 / \mathrm{T}$ law of paraelectric material, shows that extrinsic free charges contribute to the overall dielectric parameters.

- The dielectric permittivity and associated losses are relaxed towards more standard values following a nearly perfect Debye process.

Both these behaviours showed that the free charges accumulate in the form of space charges. Unlike the case of single crystals for which the space charges accumulate at the crystal surface, the space charges are stored at the grain boundaries in core-shell composites. Silica then acts as a dielectric barrier to block the long range motion of the free charges. To further confirm that the colour and dielectric changes were to be ascribed to $\mathrm{Ti}^{3+}$ centers, temperature dependent electron paramagnetic resonance was undertaken. The thermal activation of the $\mathrm{Ti}^{3+}$ EPR line was shown to follow the same Arrhenius law as the dielectric one with a common activation energy of $100 \mathrm{meV}$ thus establishing a link between macroscopic and microscopic spectroscopy techniques. $\underline{21}$ In agreement with early computations, such activation energy coincides with the $\mathrm{Ti}^{3+}$ impurity level in the band gap of $\mathrm{BaTiO}_{3}$ just beneath the bottom of the conduction band. $\underline{22}$ This is thus clear evidence that the colour and the effective dielectric permittivity can be associated with $\mathrm{Ti}^{3+}$ defects.

\subsection{Correlation between macroscopic and local properties}

It is a very general rule that macroscopic experiments (including the naked eyes' one) should be supplemented by local spectroscopy techniques and confirmed by ab initio electronic computations to clarify the origin of extrinsic functionalities. 
A very good example of this is the contribution of charged defects to the optical properties of ferroelectric single crystals. For decades, $\mathrm{BaTiO}_{3}$ single crystals contained native charged defects like $\mathrm{Fe}^{3+/ 2+}$ on the $\mathrm{Ti}^{4+}$ site in the 100 atomic ppm range, which is far too large for their reliable use under high power laser radiation. Only when this unwanted defect level could be brought to the sub-10 ppm range could the influence of controlled defects (like $\mathrm{Co}^{4+}$ ) be investigated. $\underline{23}$

Electron Paramagnetic Resonance (EPR) is a very useful tool in this process to correlate linear and nonlinear optical properties with the intentionally introduced charged defects. $\frac{24}{}$ Emphasis was laid on polaron formation linking the electronic excitations needed for large non-linear coefficients with the lattice polarizability, which is a key figure of ferroelectrics. In the case of the blue $\mathrm{BaTiO}_{3}$ ceramics already mentioned, temperature dependent $\mathrm{Ti}^{3+}$ centres which are very active in EPR were related to the giant overall dielectric parameters. $\underline{20,24}$ Because of its unique detection ability, EPR was also successfully used in thin films, showing that $\mathrm{Ti}^{3+}$ polarons lead to low temperature dielectric relaxation similar to the one reported a long time ago in bulk perovskites. $\frac{25-27}{}$

Extended X-ray Absorption Fine Structure (EXAFS) has also long been applied to ferroelectric materials. $\underline{28}$ Local off-centring of even isovalent impurities has been shown to contribute largely to the dielectric permittivity and its dispersion dynamics versus frequency. Again unwanted impurities of polaron nature could contribute to a polaron-like feature seen by EXAFS in high purity single crystals like $\mathrm{SrTiO}_{3} . \underline{29}$

Obviously nowadays atomic resolution which has been reached using High Resolution Transmission Electron Microscopy (HRTEM) and its chemically sensitive counterpart EELS can be used to discriminate point defects at very microscopic scales. However, resolving a small amount of point defects uniformly spread in a sample is still a matter of debate, all the more, when oxygen vacancies are involved.

\subsection{Use of acceptor centres for electron trapping}

Once ascertained and documented, the occurrence of unwanted oxygen vacancies can be detrimental for the reliable use of ferroelectric materials. While unavoidable, their effect on dielectric properties, which mostly increases the losses, may be overcome.

Taking into account that the main contribution of oxygen vacancies on losses and conductivity is to provide electrons, which can hop up on the shallow $\mathrm{Ti}^{3+}$ levels, one has to trap these electrons. This is achieved by using acceptor centres, the most well-known being $\mathrm{Mn}^{4+}$ at the $\mathrm{Ti}^{4+}$ sites. Since the reduction of $\mathrm{Mn}^{4+}$ to $\mathrm{Mn}^{3+}$ takes more energy than the $\mathrm{Ti}^{4+} / \mathrm{Ti}^{3+}$ process, the electron trapping will be more efficient and the conductivity will be reduced. Such deeper trapping levels are efficient, which explains why they are considered for many of the applications of bulk ferroelectrics.

This has been transferred to thin films for which the residual conductivity is even more critical since the electric fields are already large at moderate voltages because of the limited thickness of the films. Leakage currents are one of the main limitations of these films mostly because of unavoidable oxygen vacancies. $\frac{30,31}{1}$ The availability of industry scale thin films allows for reliable processing and for statistical data handling. On substituting $\mathrm{Mn}^{4+}$ for $\mathrm{Ti}^{4+}$ up to 1 atm\% the Fermi level could be lowered in the band gap ( $\underline{\text { Fig. } 5}$ ) thus showing that the impurity level close to the conduction bands has less influence. $\frac{32}{}$ As a consequence charge injection from the top electrode which was possible in pure thin films was greatly reduced and the leakage current decreased by more than three orders of magnitude. Such concurrent in depth XPS which leads to the electronic scheme of Fig. 5 and leakage current measurement are not so easy in bulk materials. Thin films thus offer case studies for the shift from Schottky injection to the PooleFrenkel conduction upon $\mathrm{Mn}$ doping. 


\section{Water related radicals as polarization probes}

After having described some of the consequences of oxygen sub-stoichiometry, we now turn to the case of additional defects which may have significant consequences. These are water related radicals which are always present under normal conditions in air. While such centres and their coupling with ferroelectric polarization may be of interest for surface photochemistry, they can obscure some experiments. $\frac{33,34}{T}$ This latter point is discussed here.

First, the total removal of such defects upon simple annealing should be ignored since bulk -OH was observed by infra-red absorption even in single crystals grown from the melt at $1500{ }^{\circ} \mathrm{C}$. Also, in nanopowders, differential scanning calorimetry evidenced a departure of $-\mathrm{OH}$ at temperatures as high as 500 ${ }^{\circ} \mathrm{C}$. This shows that, in nanostructured materials like nanopowders or polycrystalline films of large specific area, $-\mathrm{OH}$ are a concern, particularly in classical soft chemistry processing.

The way water can disturb experiments is apparent in local probe microscopies like Atomic Force Microscopy (AFM) or Piezo Force Microscopy (PFM). In these, a tip is scanned over the surface, while a bias is applied between the tip and the surface leading to an extremely large local electric field. It is now well recognized that the full removal of adsorbed water is a necessary condition for reliable AFM and PFM experiments because these charges may move under the tip.

The way $-\mathrm{OH}$ can be trapped in ferroelectric oxides is three fold: chemically inserted in oxygen vacancies in the bulk, chemisorbed onto the surface or physisorbed on the charged surface. This last process is of most interest to physicists because it may result from a direct Coulomb interaction between the charged radical and the ferroelectric polarization charges. It can help in screening the polarization charges, thus decreasing the depolarization electric field and reducing the density of ferroelectric domains. Even the critical size for the stabilization of a ferroelectric state could be changed depending on the amount of physisorbed $-\mathrm{OH}$.

Obviously, before checking the influence of physisorbed radicals, one has to remove all the extrinsic $-\mathrm{OH}$ whether bulk substituted or surface chemisorbed, not an easy task, as already recalled. The way towards such a removal is to better understand and control their contribution during the nucleation and growth of ferroelectric nanoparticles. This was achieved using the supercritical fluid technology route where the $\mathrm{Ba}, \mathrm{Sr}$ and $\mathrm{Ti}$ precursors react in a supercritical water/ethanol mixture. $\frac{34-36}{}$ There, the fluid behaves simultaneously like a gas and a liquid thus enabling accurate control of the nucleation rate using temperature and pressure as the driving parameters. In doing so, the full solid solution $\mathrm{Ba}_{1-\mathrm{x}} \mathrm{Sr}_{\mathrm{x}} \mathrm{TiO}_{3}(0 \leq \mathrm{x}$ $\leq 1$ ) can be achieved while keeping the average grain size well below $20 \mathrm{~nm}$ and removing the bulk $-\mathrm{OH}$ to a great extent. The main result seen is that the density of surface $-\mathrm{OH}$ observed through infra-red absorption and $\mathrm{X}$-ray photoelectron spectroscopy, the average grain size and the ferroelectric properties are interrelated (ig. 6).

At this stage, it is thus clear that surface $-\mathrm{OH}$ takes part in the growth kinetic as well as screens the ferroelectric polarization. Segregating between chemisorption and physisorption thus remains to be achieved.

\section{Solid solutions for tuning ferroelectric properties}

The last part of this short review is about heavy substitution in ferroelectric compounds and its consequences on the ferroelectric and piezoelectric properties. 
Indeed, the quest for improved functionality requires new ferroelectric compounds while the range of possibilities is necessarily restricted. On scanning the binary and ternary solid solutions, chemists and material scientists should not act randomly. One example of such sensible research can be the ternary compound $\mathrm{BaTiO}_{3}-\mathrm{CaTiO}_{3}-\mathrm{BaZrO}_{3}$ (BCTZ) where, starting from ferroelectric $\mathrm{BaTiO}_{3}$, ferroelectricity is suppressed by full substitution on both cationic sites. In this triangular phase diagram (Fig. 7), the initial aim was to understand how the ferroelectricity was gradually transformed into a relaxor non-ferroelectric

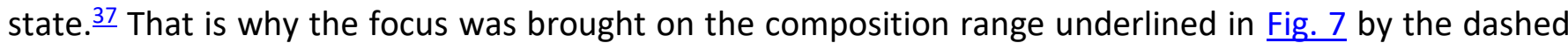
area. This means that a vast majority of long lasting experiments were focused on this border area. The main result was continuity between ferroelectricity and relaxor as the disorder increases when moving from ferroelectric $\mathrm{BaTiO}_{3}$ down to $\mathrm{BaZrO}_{3}-\mathrm{CaTiO}_{3}$.

Such behaviour is in line with many $\mathrm{BaTiO}_{3}$-based solid-solutions and it can fit the polarizability model in which the local disorder (so-called "breather" mode) is injected in a long range ferroelectric lattice. $\frac{38,39}{}$ This very basic concern is of major interest for applications. Indeed, a more recent research on the same BCTZ ternary diagram was, by chance, focused on the same composition range where the disorder starts acting macroscopically. $\underline{40}$

Following the dashed line drawn in Fig. 7, the authors were able to record extremely large piezoelectric coefficients which can even compete with those of $\mathrm{PbTiO}_{3}-\mathrm{PbZrO}_{3}(\mathrm{PZT})$, which is a reference. Unlike the former cases for which disorder arises from the charge imbalance resulting in free charge motion, full substitution in BCTZ induces polar disorder without long range ionic motion. The intermediate length scale of polarization disturbance resulting from $\mathrm{Zr}^{4+} / \mathrm{Ti}^{4+}$ and $\mathrm{Ca}^{2+} / \mathrm{Ba}^{2+}$ substitutions breaks down the $\mathrm{BaTiO}_{3}$ net polarization. This induces the gradual cross-over from the ferroelectric to the relaxor state. This also gives rise to a large number of equivalent orientations for the polarization which starts to be highly flexible under minor external stresses. $\frac{40}{}$ Such a picture is consistent with the quadruple critical point resulting from the convergence of all the ferroelectric transition lines for the composition $\mathrm{Ba}_{0.89} \mathrm{Ca}_{0.11} \mathrm{Ti}_{0.87} \mathrm{Zr}_{0.13} \mathrm{O}_{3}$ (Fig. 8). .11

The occurrence of multiple equivalent polarization states is intended to provide the necessary flexibility for increased polarization performance. While piezoelectric coefficients in lead-free solid solutions are really competing with those of PZT, the main issue is that the transition temperature remains low and thus does not allow for stable and long lasting polarization in ceramics operating at room temperature. $\underline{42}$ This is precisely the main drawback of BCTZ whose best composition in the convergence range has a ferroelectric transition very close to room temperature.

\section{Conclusion}

To summarize, the occurrence of charged defects and the control of stoichiometry in ferroelectric materials are still topics of interest. The challenges at the synthesis stage are increasing as the size of materials decreases to comply with the needs of applications. At the same time, advanced modelling of ferroelectric and related compounds also requires the highest possible control of defects.

\section{Notes and references}

1. E. Duverger, B. Jannot, M. Maglione and M. Jannin, Solid State Ionics, 1994, 73, 138.

2. N. H. Chan, R. K. Sharma and D. M. Smyth, J. Am. Ceram. Soc., 1981, 64, 556-562.

3. D. M. Smyth, Solid State Ionics, 2000, 129, 5-12.

4. M. Vollman and R. Waser, J. Am. Ceram. Soc., 1994, 77, 235-243.

5. R. Waser, Ferroelectrics, 1992, 133, 109. 
6. D. M. Smyth, J. Electroceram., 2002, 9, 179-186.

7. F. D. Morrison, A. M. Coats, D. C. Sinclair and A. R. West, J. Electroceram., 2001, 6, $219-232$.

8. R. Stumpe, D. Wagner and D. Bäuerle, Phys. Status Solidi A, 1983, 75, 143.

9. R. Waser and M. Klee, Integr. ferroelectr., 1992, 2, 23.

10. O. Bidault, P. Goux, M. Kchikech, M. Belkaoumi and M. Maglione, Phys. Rev. B: Condens. Matter, 1994, 49, 7868-7873.

11. R. Waser, J. Nanosci. Nanotechnol., 2012, 12, 7628-7640.

12. H. Kohlstedt, A. Petraru, K. Szot, A. Rüdiger, P. Meuffels, H. Haselier, R. Waser and V. Nagarajan, Appl. Phys. Lett., 2008, 92, 062907.

13. R. Waser, R. Dittmann, T. Gstaikov and K. Szot, Adv. Mater., 2009, 21, 2632-2663.

14. M. P. Singh, L. Méchin, W. Prellier and M. Maglione, Appl. Phys. Lett., 2006, 89, 202906.

15. S. Komornicki, J. C. Grenier, M. Pouchard and P. Hagenmuller, New J. Chem., 1981, 5, 161-166.

16. D. C. Meyer, A. A. Levin, T. Leisegang, E. Gutmann, P. Paufler, M. Reibold and W. Pompe, Appl. Phys. A, 2006, 84, 31-35.

17. E. Arveux, S. Payan, M. Maglione and A. Klein, Appl. Surf. Sci., 2010, 256, 6228-6232.

18. Y. Kobayashi, O. J. Hernandez, T. Sakaguchi, T. Yajima, T. Roisnel, Y. Tsujimoto, M. Morita, Y. Noda, Y. Mogami, A. Kitada, M. Ohkura, S. Hosokawa, Z. Li, K. Hayashi, Y. Kusano, J. Kim, N. Tsuji, A. Fujiwara, Y. Matsushita, K. Yoshimura, K. Takegoshi, M. Inoue, M. Takano and H. Kageyama, Nat. Mater., 2012, 11, 507-511.

19. S. Mornet, C. Elissalde, V. Hornebecq, O. Bidault, E. Duguet, A. Brisson and M. Maglione, Chem. Mater., 2005, 17, 4530-4536.

20. U.-C. Chung, C. Elissalde, S. Mornet, M. Maglione and C. Estournès, Appl. Phys. Lett., 2009, $94,072903$.

21. A. Artemenko, C. Elissalde, U.-C. Chung, C. Estournès, S. Mornet and M. Maglione, Appl. Phys. Lett., 2010, $97,132901$.

22. M. Actis and F. Michel-Calendini, Int. J. Quantum Chem., 1997, 61, 657-664.

23. D. Rytz, A. Wechsler, M. H. Garrett, C. C. Nelson and R. N. Schwartz, J. Opt. Soc. Am. B, 1990, 7, 2245.

24. E. Possenriede, P. Jacobs and O. F. Schirmer, J. Phys.: Condens. Matter, 1992, 4, 4719-4742.

25. V. V. Laguta, A. M. Slipenyuk, I. P. Bykov, M. D. Glinchuk, M. Maglione, D. Michau, J. Rosa and L. Jastrabik, Appl. Phys. Lett., 2005, 87, 022903.

26. A. Artemenko, S. Payan, A. Rousseau, D. Levasseur, E. Arveux, G. Guegan and M. Maglione, AIP Adv., 2013, 3, 042111.

27. O. Bidault, M. Maglione, M. Actis, M. Kchikech and B. Salce, Phys. Rev. B: Condens. Matter, 1995, 52, 4191.

28. J. Mustre, Y. Yacoby, E. A. Stern and J. J. Rehr, Phys. Rev. B: Condens. Matter, 1990, 42, 10843.

29. M. Fischer, A. Lahmar, M. Maglione, A. San Miguel, J. P. Itie, A. Pollian and F. Baudelet, Phys. Rev. B: Condens. Matter, 1994, 49, 12451.

30. E. Bouyssou, R. Jerisian, N. Cezac, P. Leduc, G. Guegan and C. Anceau, Mater. Sci. Eng., B, 2005, 118, 2833.

31. M. Dawber, K. M. Rabe and J. F. Scott, Rev. Mod. Phys., 2005, 77, 1083.

32. D. Levasseur, E. Bouyssou, R. De Paolis, A. Rousseau, F. Coccetti, G. Guegan, S. Payan and M. Maglione, J. Phys.: Condens. Matter, 2013, 25, 495901.

33. J. Kreisel, M. Alexe and P. A. Thomas, Nat. Mater., 2012, 11, 260-260. 
34. J. L. Wang, B. Vilquin and N. Barrett, Appl. Phys. Lett., 2012, 101, 092902.

35. G. Philippot, K. M. O. Jensen, M. Christensen, C. Elissalde, M. Maglione, B. B. Iversen and C. Aymonier, J. Supercrit. Fluids, 2014, 87, 111-117.

36. G. Philippot, C. Elissalde, M. Maglione and C. Aymonier, Adv. Powder Technol., 2014, 25, 1415-1429.

37. J. Ravez, C. Broustera and A. Simon, J. Mater. Chem., 1999, 9, 1609.

38. A. Simon, J. Ravez and M. Maglione, J. Phys.: Condens. Matter, 2004, 16, 963.

39. A. R. Bishop, A. Bussmann-Holder, S. Kamba and M. Maglione, Phys. Rev. B: Condens. Matter, 2010, 81, 064106

40. W. Liu and X. Ren, Phys. Rev. Lett., 2009, 103, 257602.

41. D. S. Keeble, F. Benabdallah, P. A. Thomas, M. Maglione and J. Kreisel, Appl. Phys. Lett., 2013, 102, 092903.

42. J. Roedel, W. Jo, K. T. P. Seifert, E. M. Anton, T. Granzow and D. Damjanovic, J. Am. Ceram. Soc., 2009, 92, 1153-1177. 


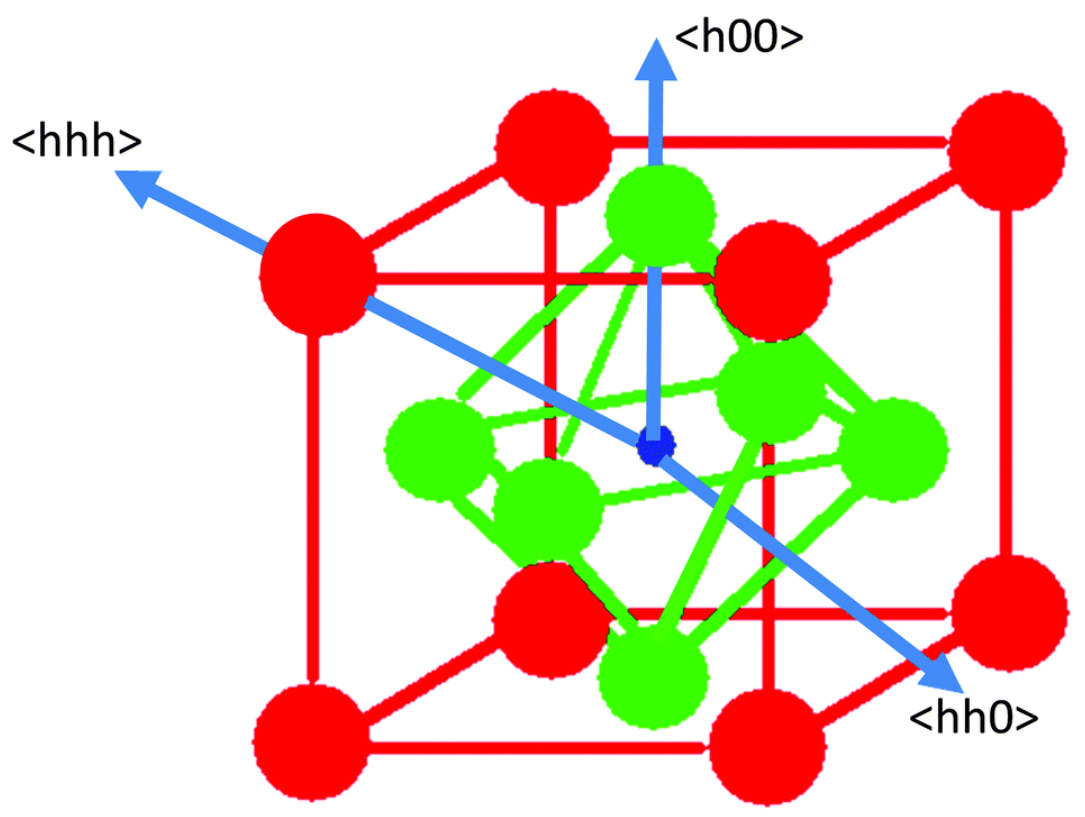

Fig. 1 Sketch of the $\mathrm{BaTiO}_{3}$ unit cell with Ti sitting at the cube center, $\mathrm{Ba}$ at the corners and $\mathrm{O}$ at the face centers. The directions along which Ti can be displaced by less than $0.2 \AA$ are one of the $6\langle h 00\rangle$, of the $12<h h 0\rangle$ and of the $8<\mathrm{hhh}>$ vectors leading respectively to tetragonal, orthorhombic and rhombohedral phases.

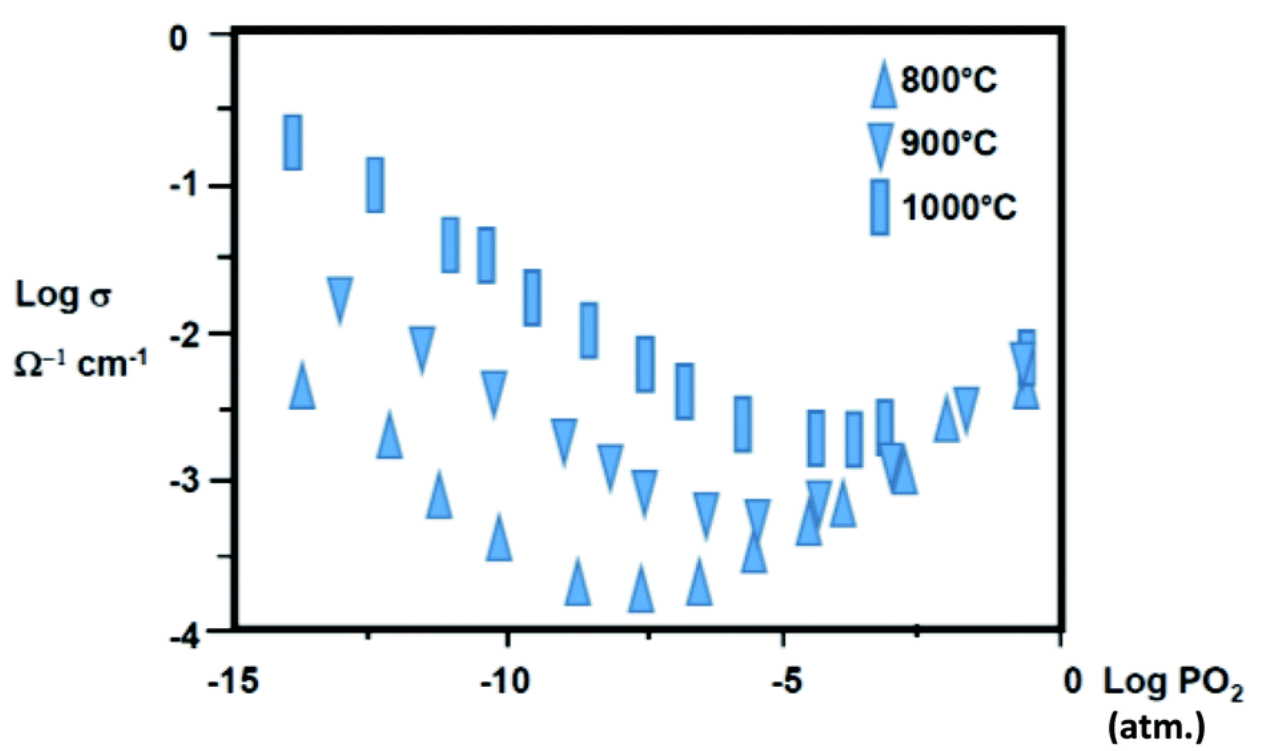

Fig. 2 Isothermal conductivity of $\mathrm{BaTiO}_{3}$ single crystal versus the oxygen partial pressure. $\frac{1}{}$ The positive slope close to the ambient partial pressure stems from hole conductivity while the negative slope under highly reducing conditions is of electronic nature. The minimum conductivity which shifts to a higher oxygen partial pressure on increasing temperature directly relates to the density of impurity defects within the band gap. $\underline{\underline{1-5}}$ 


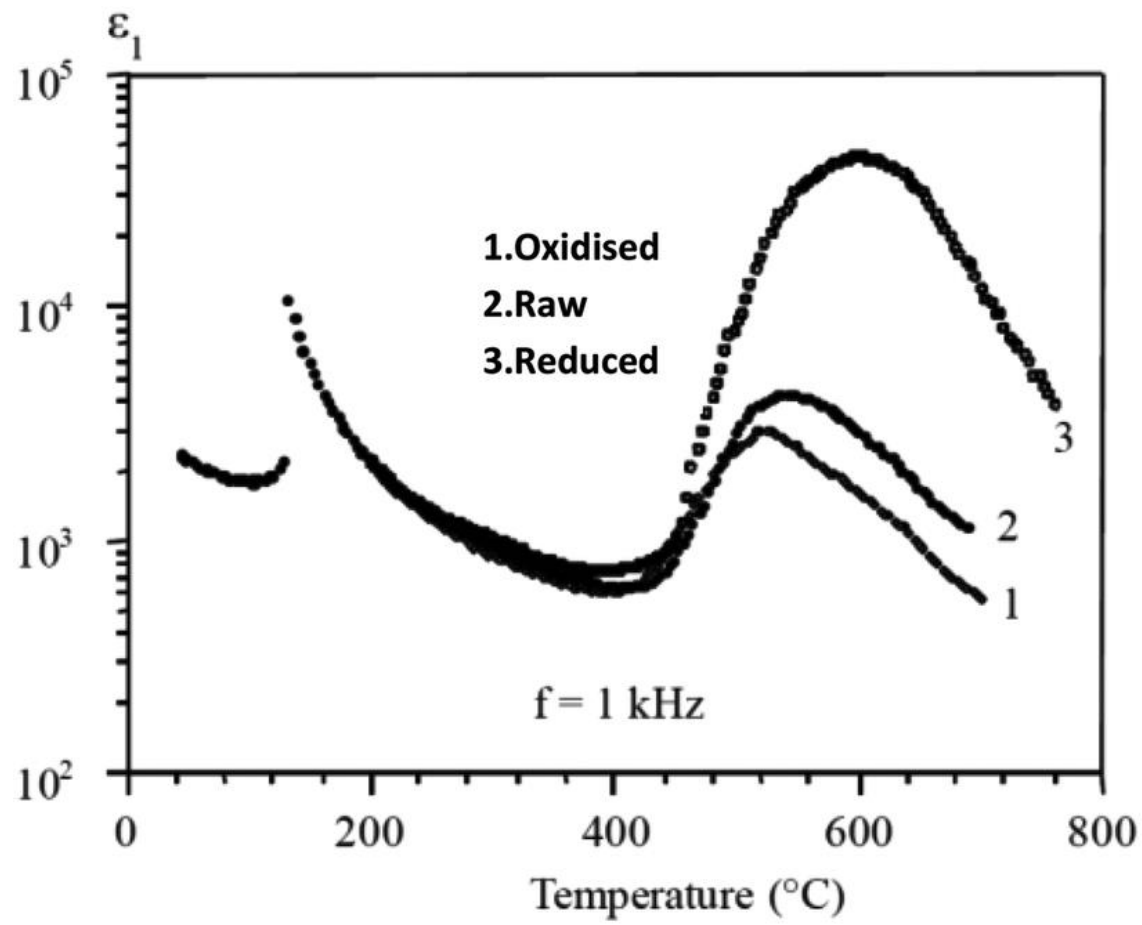

Fig. 3 Dielectric permittivity of raw, reduced and re-oxidised $\mathrm{BaTiO}_{3}$ single crystal from the same starting batch. The broad and large dielectric anomaly at high temperatures is clearly related to the amount of charged defects, while the ferroelectric transition is undisturbed. Note the log scale for permittivity which shows extremely large values which were explained in terms of space charge accumulation.
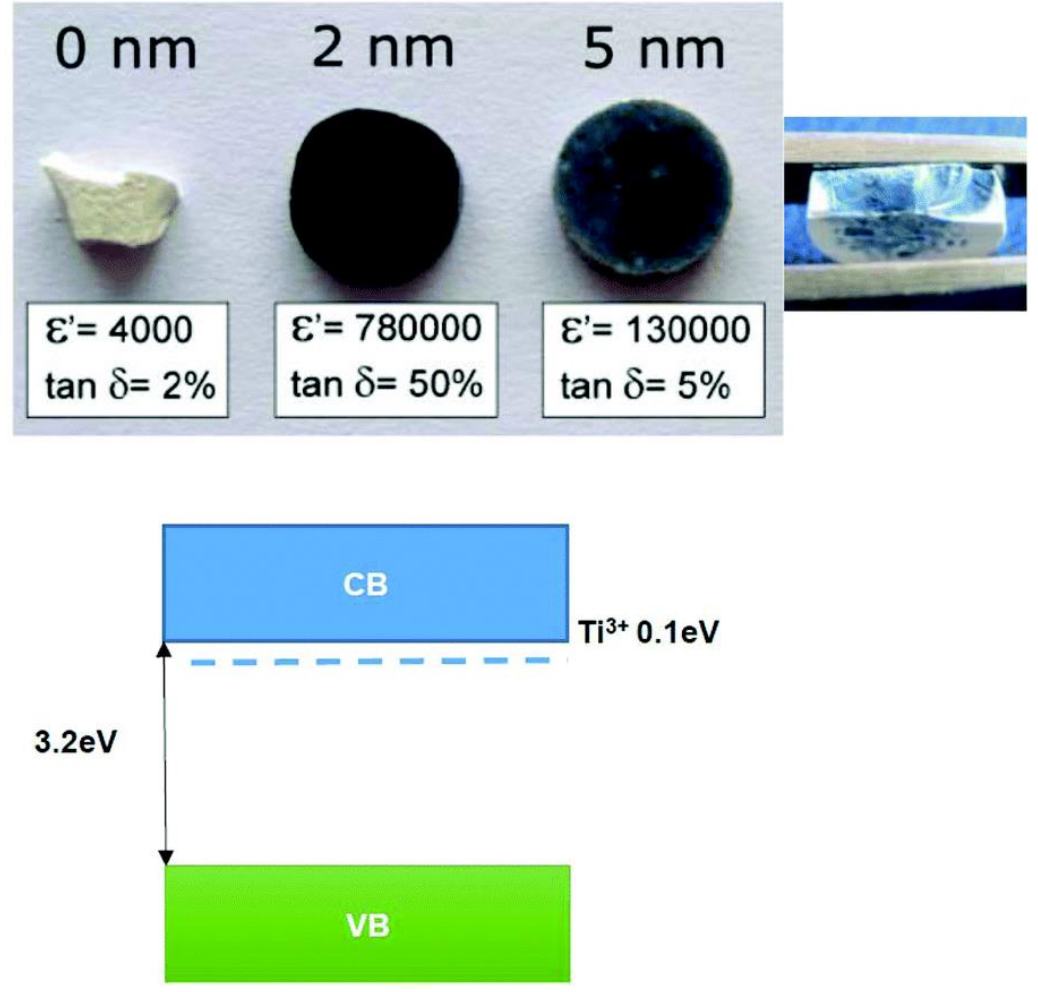

Fig. 4 Colour tuning of $\mathrm{BaTiO}_{3} @ \mathrm{SiO}_{2}$ core-shell particles sintered under a reducing atmosphere by spark plasma sintering. White pellets are made of uncoated $\mathrm{BaTiO}_{3}$ grains and the darkest pellets include the thickest $\mathrm{SiO}_{2}$ shell of $5 \mathrm{~nm}$. The effective dielectric permittivity and losses at room temperature and for a frequency of $100 \mathrm{kHz}$ are given for each pellet. Inset is a schematic description of the $\mathrm{Ti}^{3+}$ impurity level within the band gap which explains 
the colour change. The occurrence of such defects in highly reduced materials was confirmed on the basis of electron paramagnetic resonance.
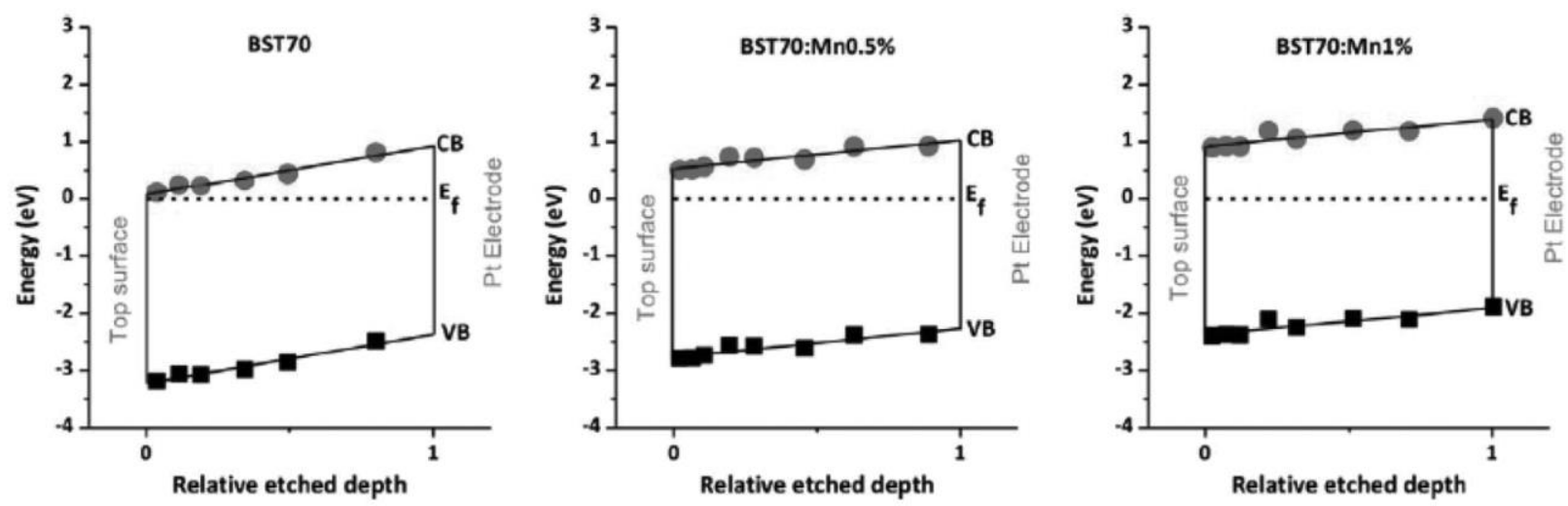

Fig. 5 Shift of the band gap of BST thin films for several substitution rates of Mn to Ti from 0 to $1 \%$. The shift was obtained through XPS spectra of the valence band on Ar-etching the films from the top surface (left of each diagram) to the substrate. The band gap was assumed to be fixed to the theoretical value of $3.2 \mathrm{eV}$ and the Fermi level was set to the one of the metallic bottom electrode.

a)

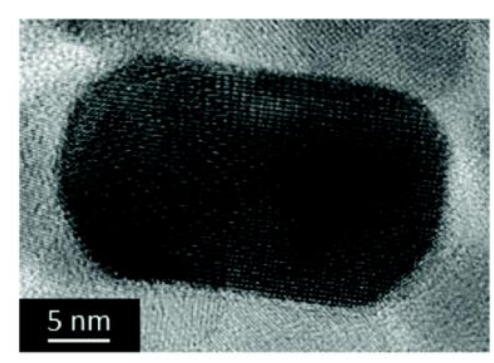

b)

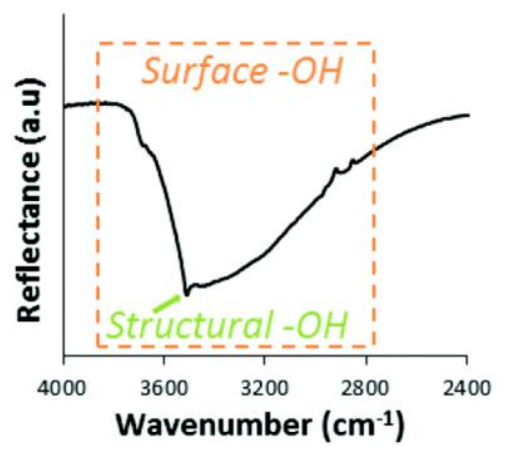

c)

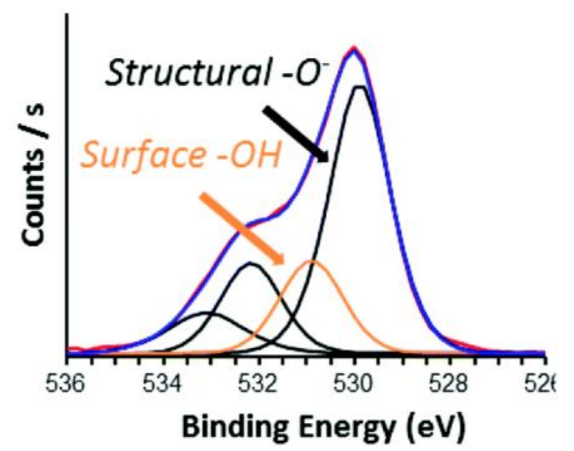

Fig. 6 Characterization of $\mathrm{BaTiO}_{3}$ nanocrystals produced using the continuous supercritical fluid technology; (a) high resolution TEM image confirming the synthesis of nanocrystals, (b) FTIR analysis highlighting the presence of both, structural and surface, $-\mathrm{OH}$ defects and (c) XPS analysis to have a semi-quantitative estimation of the surface $-\mathrm{OH}$ amount. 


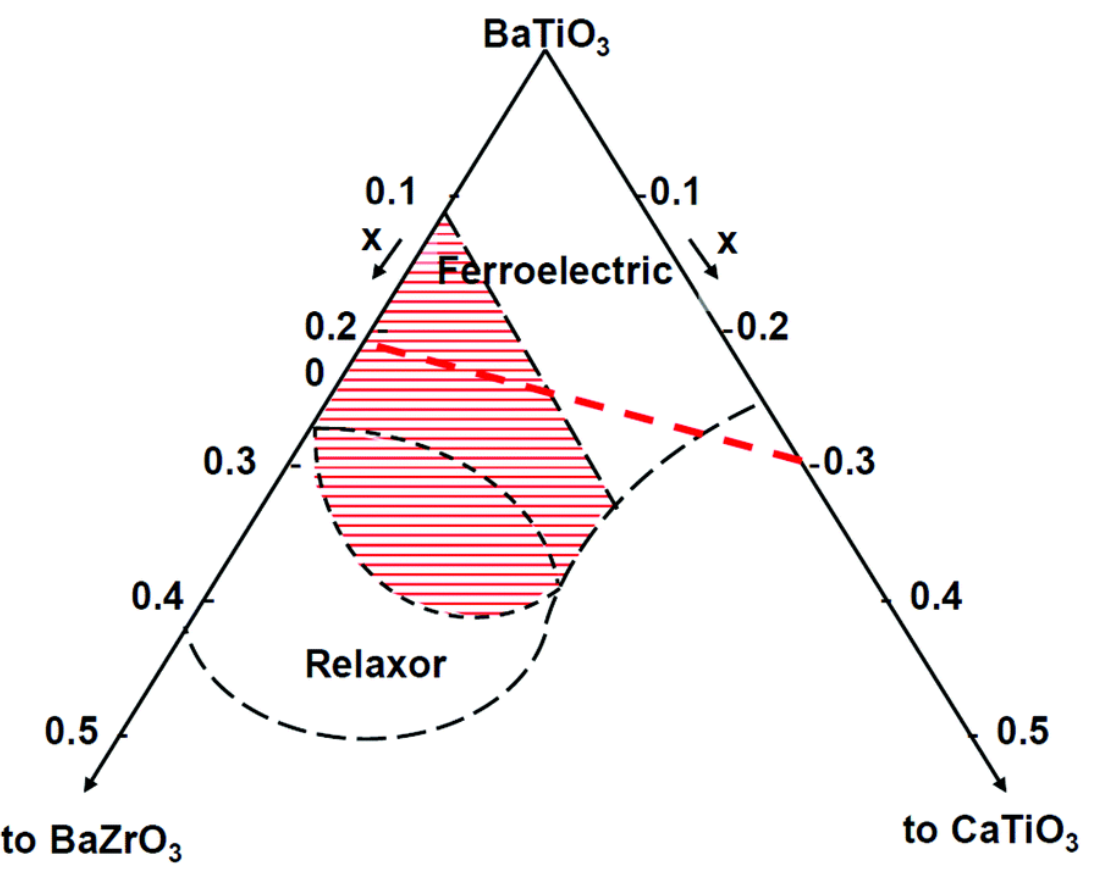

Fig. 7 Ferroelectric phase diagram of the ternary $\mathrm{BaTiO}_{3}-\mathrm{CaTiO}_{3}-\mathrm{BaZrO}_{3}(\mathrm{BCTZ})$ solid-solution. In the intermediate composition range a gradual cross-over from ferroelectric to relaxor was observed (dashed area). It is also in this intermediate range that extreme piezoelectric coefficients were reported along the dashed line.

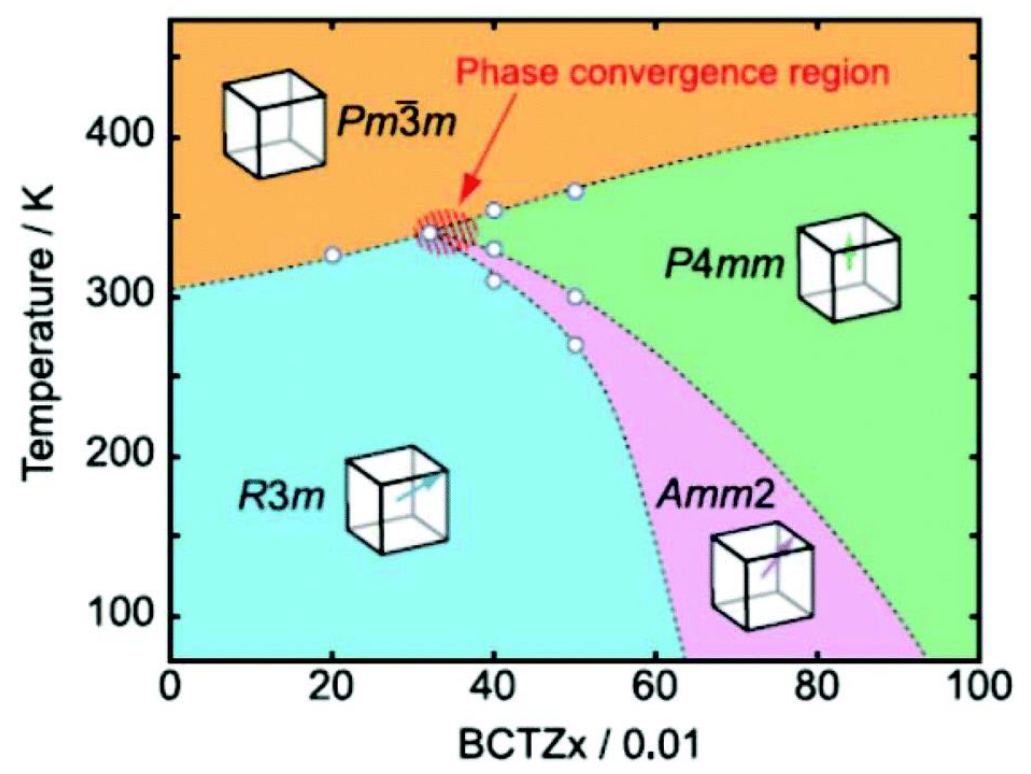

Fig. 8 Structural phase diagram of $\mathrm{BaTiO}_{3}-\mathrm{CaTiO}_{3}-\mathrm{BaZrO}_{3}$ along the dashed line of Fig. 7. The compositions were scanned under increasing $x$ in the formula $x\left(\mathrm{Ba}_{0.7} \mathrm{Ca}_{0.3} \mathrm{TiO}_{3}\right)-(1-\mathrm{x})\left(\mathrm{BaZr}_{0.2} \mathrm{Ti}_{0.8} \mathrm{O}_{3}\right)$. The largest piezoelectric coefficients were recorded in the convergence composition range for $x$ close to 0.3 . 